


\title{
ANÁLISIS DE LA BÚSQUEDA ASTROBIOLÓGICA DE INTELIGENCIA DESDE LA PERSPECTIVA DE LA MENTE CONSCIENTE
}

\author{
Una reformulación de la Ecuación de Drake \\ por \\ Henry Llanos Chilet
}

Resumen: La llamada "búsqueda de vida" fuera de la tierra en la que se basa la exobiología, parte importantísima de la astrobiología, encierra en realidad la búsqueda de la mente consciente en otras partes del universo, pues se trata implícitamente de buscar "nuestro similar". Esta idea de "nuestro similar" ha tomado muchos enfoques a lo lago de la historia e implica la búsqueda incansable de parte de filósofos y científicos de la cualidad que nos define como seres humanos y personas. En este trabajo haremos un breve recuento de tales búsquedas hasta llegar al concepto de mente consciente y expondremos lo que sabemos hasta ahora de cómo, en el caso de la Tierra, apareció esta singular cualidad: la consciencia. Luego intentaremos extrapolar este conocimiento fuera de la Tierra, lo que yo llamo "paradigma geocéntrico", en una reformulación de la conocidísima Ecuación de Drake. Identificaremos los tres cuellos de botella o eventos improbabilísimos que fueron necesarios que sucediesen para el arribo de la mente consciente en este planeta y por ende de la humanidad. Suponemos que esa misma secuencia de eventos improbables es necesaria para que, en otros planetas, halla seres similares a nosotros con consciencia.

Palabras clave: Ecuación de Drake, consciente, mente, mente consciente, neurona, pluricelularidad, vida. 
Sobre el autor. Henry Llanos Chilet es Bachiller en ingeniería de sistemas y egresado de la Maestría de Filosofía de la Ciencia de la Universidad Nacional Mayor de San Marcos - Perú. Presidente de la Asociación Peruana de Ateos - APERAT. Miembro del Instituto de Extrapolítica y Transhumanismo (IET).

Información de contacto: henry.llanos@gmail.com

Imagen de portada. Microfotografía electrónica de barrido en falso color de los pelillos terminales de los dedos tomada por Luis Monje.

Llanos, H. (2021). Análisis de la búsqueda astrobiológica de inteligencia desde la perspectiva de la mente consciente. Una reformulación de la Ecuación de Drake. Instituto de Extrapolítica y Transhumanismo, 4(1), https://doi.org/10.52749/iet.v4i1.1

(C) Sociedad Secular Humanista del Perú (SSH).

Fondo Editorial de la Sociedad Secular Humanista del Perú.

Director de Revista: Piero Gayozzo

Publicaciones del Instituto de Extrapolítica y Transhumanismo (IET) de la SSH.

Correo: extrapolítica@ssh.org.pe

Esta obra está bajo licencia internacional Creative Commons 4.0. 

DE LA MENTE CONSCIENTE

\section{Introducción}

La tendencia del humano de buscar "su similar" fuera de la Tierra surgió casi después de la revolución copernicana, cuando vemos a Giordano Bruno imaginando cada estrella como otros solescon mundos similares al nuestro (CAPPELLETTI, 1981). Esta tendencia, sin embargo, empezó a configurarse desde los albores de la humanidad cuando nuestros antepasados separaban a los objetos que observaban en dos categorías intuitivas, animados e inanimados, tal vez teniendo en cuenta que unos se movían por sí solos y los otros no.

A medida que la civilización se instaura, vemos a Aristóteles preocupándose seriamente sobre el tema de los seres vivos y de los seres racionales como el hombre. No es sino muchos siglos después, con Descartes, donde el asunto de la mente consciente se formaliza en el ámbito académico con la introducción del concepto definido del "yo" (Ponzio, 2006).

Llegada una mejor comprensión del fenómeno de la vida con la revolución darwiniana y posteriormente con la genética, aunado a la confirmación de la tesis de la pluralidad de los mundos por el desarrollo de la astronomía (todo esto entre los siglos XIX y XX), la discusión sobre la búsqueda de nuestros similares fuera de la tierra se centra en el concepto de vida. Posteriormente la revolución informática, iniciada con el asunto de la inteligencia artificial por Alan Turing, trae por primera vez a colación el concepto de inteligencia como una característica que nos distingue del resto de las especies vivas. Se considera que buscar a nuestros similares no es solo buscar vida, si no también buscar inteligencia. La ecuación formulada por Drake y el proyecto Seti son claros ejemplos de ello y empieza a hablarse de comunidades tecnológicas.

Pero el concepto de mente consciente, que a mi juicio es el más importante para todos estos fines, fue dejado de lado en la búsqueda extraterrestre. Tal vez porque se mantuvo sólo en el plano de la filosofía desde Descartes, hasta que quiso repuntar tímidamente en el escenario médico con la llegada del psicoanálisis de Freud, para volverse a esconder por las innumerables críticas hacia él. 0 tal vez por el excesivo optimismo que se puso en la inteligencia artificial como generador de otro ser similar a nosotros (a mitad del siglo XX) sin haberlo logrado hasta ahora.

Sin embargo, después de los grandes avances neurocientíficos de las últimas décadas y de la instauración de la filosofía de la mente como nuevo campo de interés en la filosofía actual, ya el concepto de mente consciente está suficientemente sólido para desplazar al concepto de inteligencia (e incluso al de vida) en la búsqueda astrobiológica. Lo que se busca en realidad son seres con mente consciente fuera de la tierra. Las cualidades de inteligencia, interés en comunicarse con el resto, voluntad (y demás) están inherentemente unidos al fenómeno de la consciencia y, de hecho, los contiene a todos.

¿Qué posibilidades tenemos que surja la mente consciente del fenómeno de la vida? ¿O tal vez pueda surgir en un ser "no vivo", con lo que podríamos creer que el fenómeno de la vida no es necesario para su surgimiento? 


\section{La búsqueda astrobiológica. Paradigma geocéntrico}

Los fenómenos de la vida, la mente consciente y la inteligencia han surgido en la Tierra siendo nuestro planeta el único caso de estudio que tenemos. De las exploraciones en el sistema solar, e incluso del espacio interestelar, sólo se han encontrado los materiales químicos básicos para la vida (de tipo terrestre) en cometas, satélites y en las nebulosas de sistemas planetarios nacientes (Bachiller, 2015). Se cree que en Marte pudo haber habido vida unicelular (Sagan, 1994), cosa por confirmar. Ningún mínimo rastro se ha encontrado, ni se espera encontrar, de los otros dos fenómenos (conciencia e inteligencia) en el sistema solar hasta donde pueden informar las sondas espaciales.

Como vemos tenemos una muestra muy pequeña de solo un caso -la Tierra- para poder hacer proyecciones acerca de la existencia, abundancia y distribución de los tres fenómenos en el universo. Hemos de aceptarlo, en su mayor parte lo que se diga al respecto será especulativo. Esto no significa, por supuesto, que no se deba hablar del tema. La misma ciencia -cuya hija, la tecnología, representa el poder actual de la humanidad- empezó en las especulaciones de los filósofos. Pero se debemos especular como aquellos iniciadores de la ciencia: siempre con un método. No divagaremos en la ignorancia y sobrenaturalidad como los seudocientíficos, ufólogos y "magufos", sino que partiremos de lo que sabemos sobre estos tres fenómenos en el contexto terrestre (que no es poco) y lo extrapolaremos hacia los confines de la galaxia. Esto no es nada descabellado, si tenemos en cuenta que las mismas leyes de la física y los mismos materiales están aquí (en la Tierra) como allá (en esos confines).

Esto formará parte de lo que llamaré "paradigma geocéntrico", que es la única manera seria de especular que tenemos. Ahora nos preguntaremos ¿Qué es, a líneas generales, lo que paso en la Tierra? ¿Cómo y bajo qué circunstancias surgieron los tres fenómenos de los que hablamos (la vida, la conciencia y la inteligencia)?

Analicemos, pues, los tres fenómenos.

\section{La vida: acumuladora de información}

Usualmente los biólogos y loa científicos, que trabajan con los denominados "seres vivos", tienen una idea muy intuitiva de lo que es la vida. Muchos de ellos -y de la gente común- se quedan muy cómodos con esa intuición inefable sobre la vida que les hace no tener problemas en diferenciar entre aquelloque está vivo de lo que no lo está. Los criterios intuitivos usados son cosas a simple vista como el movimiento propio o la expresión de emociones. Sin embargo -y como era de esperarse- algunos filósofos y científicos han intentado profundizar en este fenómeno y definir a la vida tratando de llegar a su "esencia" (Bernardo, 2007). Esta tarea está muy relacionada al cómo surgió la vida y bajo qué circunstancias, que es precisamente lo que nos compete en este análisis.

En un entorno cualquiera existe un nivel de complejidad o información predeterminado. Por el devenir o el azar hay fluctuaciones de estos niveles de complejidad, pues todo en el universo siempre 
es un equilibrio dinámico, nunca estático. Esas fluctuaciones hacen que aparezcan temporalmente cuerpos con estructuras más complejas que el predeterminado del entorno.

La segunda ley de la termodinámica hace que todo tienda al punto de equilibrio -o de máximo desorden- produciendo la desaparición estas estructuras más complejas. De allí su naturaleza temporal.

En algunos de estos cuerpos temporales -más complejos- de pronto aparecen procesos que tienden a conservar su estructura compleja, haciendo que su desaparición demore en llegar. Esto sólo puede pasar cuando hay una fuente de energía externa, ya que "darle la contra" a la segunda ley requiere gastar energía para no violarla. A muchos de estos procesos, que han pasado un cierto umbral de complejidad y tienen condiciones específicas, los hemos llamado "vida". Y a los cuerpos que los poseen, "seres vivos". La fuente de energía que se ha usado en la Tierra para sostener la vida es la de la estrella más cercana, el Sol (Schrodinger, 2005).

De todo esto se deduce que el problema y función principal de la vida es conservar la estructura química interna de los cuerpos vivos, en un entorno que tiende a deshacerla. Cualquier definición que se intente hacer de lo que es "vida" debe contener esta cualidad esencial.

Otra cualidad fundamental de los procesos de la vida es que están sumergidos en un bucle de realimentación positiva hacia una mayor complejidad de los cuerpos vivos. Esto es lo que se conoce como "Evolución Biológica" y es el fenómeno descubierto por Darwin y Wallace. Los procesos de la vida que conservan la complejidad en los cuerpos vivos requieren energía útil (exergía) del entorno. Parte de esta energía útil está contenida en recursos específicos usados como materia prima y dependen del tipo de estrategia que sigue un proceso vivo específico: así una mariposa monarca necesitara como recurso el polen de las flores de acleptia, un gusano de seda, las hojas de la mora, etc.

Pero cualquier entorno tiene dichos recursos de forma limitada. Esto genera una feroz competencia entre los cuerpos vivos que usan dicho recurso y, cuando se acaba, es necesario muchas veces cambiar de estrategia. Mientras la fuente general de exergía exista -el sol en nuestro caso- los continuos cambios de estrategias hacen que los procesos de la vida se vuelvan cada vez más complejos. Se pone en marcha la evolución que, a través de las generaciones y actuando sobre poblaciones de seres vivos (nunca sobre cuerpos individuales), aumenta la complejidad de estos (Dawkins, 2009).

En efecto la evolución biológica no actúa sobre individuos sino sobre grupos de éstos o poblaciones. Por ello es necesaria la temporalidad de éstos -es decir que "envejezcan" y mueran- para ser sustituidos por otros individuos similares, nunca iguales. Este proceso de sustitución se hace a través de la copia del material genético y lo hemos llamado "reproducción". Los procesos de "copiado" del material genético no pueden ser perfectos: los hijos no deben ser iguales a los padres sino sólo similares, de lo contrario el bucle de realimentación positiva hacia la mayor complejidad no se daría y no habría evolución. La reproducción sexual es una de las formas más idóneas para que se dé la evolución, pues es una fuente rica de copiados imperfectos y estrategias competitivas de apareamiento (Dawkins, 1993). De allí que haya producido a los cuerpos más complejos que existen: el orden de los mamíferos. 
En conclusión, la vida implica dos procesos:

1) Un proceso "estático" a nivel del individuo (o cuerpo vivo) que tiende a conservar su estructura química interna inalterada o dentro de un rango de condiciones, frente a un entorno que intenta deshacerla. A este proceso lo llamaremos "gestión de la vida". Los procesos metabólicos son ejemplo de ello.

2) Un proceso "dinámico" a nivel de las poblaciones de individuos que tiende a la complejidad de éstos a través de la continua sustitución de los individuos. A este proceso se le llama "evolución biológica". Es decir, la vida tiende a acumular información (Schrödinger).

En nuestro planeta los procesos de la vida han elegido un elemento químico como base: el carbono. Hasta donde sabemos, el carbono es especial porque es el único elemento capaz de formar cadenas enormes de átomos: tanto enlazándose entre carbonos como con otros elementos (Wade, 2012). No es descabellado creer que, si hay vida en otros planetas, la mayoría debería estar formada con carbono como elemento estructural. Ningún otro elemento, ni siquiera el silicio, tiene las extraordinarias capacidades del Carbono (Guillermo Gonzalez, 2006).

\section{La mente consciente: una estrategia para gestionar la vida}

Concentrémonos ahora en los diferentes procesos y estrategias que usan los seres vivos para mantenerse con vida. Habíamos dicho que la evolución hace que, con el paso del tiempo, los cuerpos vivos se complejicen. Esto genera que los procesos de gestión de la vida también tengan que complejizarse ¿Por qué?

Para comprenderlo usemos una analogía: Administrar una microempresa sólo requiere de una organización tipo militar con un único jefe que controla a sus pocos empleados. Pero administrar una gran empresa transnacional, requiere de una estructura de control y planeamiento mucho más compleja con diferenciación en tareas especializadas. El asunto se vuelve tan complejo que fue necesaria la creación de una nueva ciencia interdisciplinar -la administración de empresas- para reunir los conocimientos y habilidades requeridas.

Así mismo, desde las primeras moléculas reproductoras (precursoras y pioneras de la vida terrestre) hasta que se concretó la primera célula procariota y luego eucariota, pasaron un sinfín de procesos y estrategias diferentes. Las formas de vida que existían durante todo este periodo de tiempo, se manejaban en el mismo nivel que los procesos bioquímicos que los sostenían. No existían las mentes (Alex de Mendoza, 2013). Pero, así como los quark y leptones se agrupan en partículas subatómicas, las que a su vez se agrupan formando átomos, los cuales se agrupan formando moléculas y las moléculas simples lo hacen -así mismo- formando macromoléculas; las células que formaban los seres unicelulares sin ninguna mente, se agruparon para formar los cuerpos multicelulares. Esto no se produjo porque seguían una finalidad, no había voluntad consciente en ellas (ni fuera de ellas) dirigiendo el devenir. Sencillamente se produjo por el mismo principio que hizo aparecer a la vida: fluctuaciones azarosas de la complejidad en un mundo que no puede estar quieto y la selección de la forma compleja (comparada con la media del entorno) que desencadena procesos que tienden a conservar dicha complejidad ganada. 
Creo que el paso a la multicelularidad, como otro nivel de agrupación más, fue el hito que permitió a los seres vivos salir del agua a tierra firme o conquistar los aires. Y lo más importante, también permitió la aparición de las mentes.

\subsection{La multicelularidad y la neurona}

Los cuerpos vivos multicelulares, al momento que gregarizaban millones o hasta billones de células que se iban diferenciando y especializando en las distintas tareas vitales, no podían seguir gestionando su vida con los mismos procesos -basados en disposiciones simples- con los que funcionan los organismos unicelulares (Alex de Mendoza, 2013). Especialmente, si tenían movimientos propios, rápidos y complejos o no estaban arraigados a un lugar como las plantas (únicos seres sin mentes conformados de billones decélulas).

Si es que seres con comportamientos complejos debían de evolucionar, se necesitaba de una unidad coordinarade movimientos que gestionara su proceso vital en su complejo cuerpo: aparecieron las neuronas y con ellas, las mentes.

Según el premio nobel de medicina de 1974, Christian de Duve, las neuronas surgieron probablemente de células eucariotas que cambiaban generalmente de forma y produjeron extensiones tubulares de su soma celular, a medida que se desplazaban como los seudópodos de las amebas actuales (prolongaciones citoplasmáticas de carácter efímero que hacen que la ameba pueda trasladarse e incorporar alimentos). Pero, en los antepasados de las neuronas, esas prolongaciones se volvieron permanentes y formaron las dendritas y los axones (Duve, 1995).

Las neuronas son células corporales -como las otras- que tienen núcleo, citoplasma, etc. Pero, según Antonio Damasio, con una diferencia de carácter funcional y otra de orden estratégico:

\section{Diferencia funcional: influyen en otras células.}

La neurona produce señales electroquímicas capaz de cambiar el estado de otras células e influir en ellas (los paramecios también usan señales eléctricas para guiar su comportamiento). Entre esas otras células a las que influyen están: 1) otras neuronas, 2) células del sistema endocrino (encargadas de secretar moléculas químicas como hormonas) y 3) células de las fibras musculares (para producir movimientos).

\section{Diferencia estratégica: referencialidad al cuerpo.}

La neurona existe en beneficio del resto de células corporales. Muchas criaturas pluricelulares como las plantas- no tienen neuronas ni las necesitan para vivir. Pero en aquellas criaturas multicelulares que tienen movimientos complejos (como todos los animales) son esenciales.

En los cerebros modestos, como el de los insectos constituidos por redes de neuronas agrupadas en ganglios, las neuronas generan emociones (fomentando la secreción de moléculas químicas) y complejos comportamientos. En los cerebros de criaturas más complejas, las neuronas -además de loanterior- llegan a imitar la estructura de partes del cuerpo a la que pertenecen. Terminan 
representando el estado del cuerpo, elaborando mapas del cuerpo para formar un sustituto virtual suyo. Permanecen conectadas al cuerpo (que representan) toda la vida, en un bucle infinito interactivo (Damasio, 2010).

\section{2. ¿Qué es una mente?: las sensaciones}

La mente surgió como un subproducto o efecto colateral de los sistemas de autorregulación propios de los seres vivos, o sea de lo que hemos llamado procesos de "gestión de la vida". Las redes neuronales pronto formaron ganglios y luego cerebros (por la naturaleza misma de las neuronas de trabajar juntas) haciéndose cargo de los procesos de gestión de la vida del cuerpo multicelular entero. Para mantener el equilibrio interno en los cuerpos vivos, los cerebros necesitan valerse de procesos de control interno y autorregulación que permanentemente estén monitoreando al cuerpo vivo. Tales procesos los Ilamaremos "Bucle interno de autorregulación y control", en adelante "BIA" (Damasio, 2010). Uno de los efectos colaterales del omnipresente BIA, al momento que el animal procesa la información sensorial externa, fueron las sensaciones.

Las sensaciones son la percepción que tiene un cerebro de los procesos de autorregulación básicos para la vida, que se suceden en el interior del cuerpo (Goldstein, 1999) y que fusionados con la actividad sensorial de los órganos de los sentidos son la materia prima de las mentes (Damasio, 2006). Las sensaciones, por tanto, surgieron como un efecto colateral del BIA y no directamente del proceso de selección natural.

Bucle Interno de Autorregulación $(\mathrm{BIA}) \rightarrow$ sensaciones $\rightarrow$ mentes

Podemos concluir diciendo que la mente la forman el discurrir (en el tiempo) de percepciones de los objetos del entorno exterior y del BIA. Es decir, la mente es el conjunto de sensaciones en un instante de tiempo (Damasio, 2010). Sostengo que las mentes son un producto inevitable de los procesos de gestión de la vida en organismos pluricelulares con movimientos y comportamientos complejos.

\subsection{La mente consciente: aparece el "Yo"}

La gran mayoría de especies vivas, incluso multicelulares con movimientos y comportamientos complejos, se las arreglan muy bien para mantenerse con vida sin tener consciencia que existen, sin un "YO". Nuevamente, para que surja el siguiente nivel de complejidad es necesario la gregarización interactiva de las unidades fundamentales y la selección natural entre las fluctuaciones de complejidad (principio visto anteriormente). Las unidades fundamentales -esta vez- serían los seres vivos pluricelulares con una mente.

En una clase filogenética de animales llamada "mamíferos", por su cerebro más grande y definido y su tendencia a gregarizarse, surgió algo excepcional: de pronto el bucle cerebro-cuerpo cuerpocerebro del BIA se hizo tan complejo que se añadió -a la mente- un proceso en donde uno se 
siente a sí mismo. Apareció un animal que -tímidamente- fue haciendo consciente sus sensaciones, al fusionar -cada vez más- los procesos sensoriales perceptivos con los procesos del BIA. De pronto empezó a percibirse y a sentirse a sí mismo (Damasio, 2010).

Estos sentimientos lograron mayor resolución con la aparición de la corteza cerebral. La cual alcanza su mayor desarrollo en los mamíferos, especialmente en el orden de los primates (LeDoux, 1999). Con la corteza cerebral se consolida definitivamente la mente consciente, sin que ello signifique que solo la corteza la produzca. La mente consciente se origina del accionar concertado de algunas zonas subcorticales y de la corteza (Damasio, 2010).

La tendencia a gregarizarse es algo fundamental para la eclosión de la consciencia y su posterior complejización. Pues es en la mamada (de animales) donde se crea un entorno más complejo de relaciones intra-específicas que requieren habilidades sociales (Waal, 2009). Las unidades sobre las que la selección natural trabajo -esta vez- fueron los animales con corteza cerebral y mente. Muchos insectos gregarios como las hormigas no desarrollaron la consciencia porque carecían de este último requisito.

Pero ¿Por qué se consolidó este tipo de mente, la mente consciente?

La consciencia, ese YO, esa subjetividad, trae consigo muchos efectos colaterales o inherentes que acentúan varias características positivas de las mentes inconscientes. Entre ellos la voluntad y la inteligencia.

\section{La inteligencia: una cualidad inherente a la mente consciente}

Sostengo que la inteligencia fue la cualidad principal por la cual la mente consciente fue positivamente seleccionada -por la selección natural- para gestionar la vida de los cuerpos más complejos. En los humanos, únicos mamíferos actuales con mentes simbólicas, la inteligencia se manifiesta en elevadas capacidades de análisis de situaciones (Houser, 2012). La voluntad, la motivación, la libertad (o su ilusión) son todos efectos colaterales de nuestro complejo cerebro con mente consciente, sobre los que no actuó la selección natural. Lo que técnicamente se llamarían "características pleiotrópicas"

Resulta evidente el valor de supervivencia de la inteligencia, definida como esa capacidad de resolver con éxito situaciones problemáticas que ofrece el entorno adverso o el grupo social mediante un análisis complejo de la situación. Por esto último, es una capacidad cognitiva por excelencia en donde parece esencial el uso de símbolos, memoria y simulación de situaciones en entornos imaginados (Zimmer, 2008).

Muchos comportamientos que parecen inteligentes, se dan en especies que no tienen mente consciente (Matej Hochel, 2002). Si esos comportamientos son genuinamente inteligentes -o noestáen debate. Para que lo sean, tendríamos que admitir que cerebros de seres como los insectos manejarían algún tipo de procesamiento simbólico lo cual no parece posible. Pero lo que si sabemos, por la moderna revolución informática del siglo pasado, es que se pueden dar comportamientos inteligentes en toda su extensión, con procesamiento simbólico y memoria, en objetos sin consciencia. 


\subsection{Inteligencia sin consciencia: zombis inteligentes}

Los estudios neurocientíficos del cerebro y la revolución informática nos llevan a pensar que inteligencia y mente consciente son dos fenómenos que podrían darse de forma separada. Esto queda muy claro en las diferentes posiciones, anhelos y reducidos logros de la Inteligencia Artificial desde la década de los 50 del siglo pasado. Jeff Hawkins, conocido más por inventar la Palm, en su libro "Sobre la inteligencia", nos da un panorama moderno de cómo podrían construirse máquinas tan inteligentes -o más- como los humanos. Hasta con autonomía propia, como en un insecto, pero sin mente consciente. Así elimina esas otras cualidades "fastidiosas" que ésta trae (voluntad, libertad, etc.) que nos hacen humanos, creando zombis inteligentes.

Dichas maquinas utilizarían, sin embargo, el mismo método o algoritmo con que la corteza cerebral humana procesa la información. Hawkins centra su atención sólo en la corteza cerebral, sabiendo deantemano lo que los neurocientíficos saben: que ella sola no es consciente (Hawkins, 2005). La mente consciente es un proceso que emerge del accionar conjunto y holístico de redes neuronales de la corteza y de muchas zonas subcorticales. Además, es posible que -sólo el caso delos humanossu mente consciente tenga extensiones físicas en su mundo cultural y social, según la teoría del exocerebro de Roger Bartra (Bartra, 2005). Algo muy plausible pues seguiría el mismo principio de gregarizacion, fluctuaciones de complejidad y selección de la forma más compleja y estable para subir al siguiente nivel de organización.

\section{Identificando los cuellos de botella}

\section{Lo que pasó en la Tierra}

Regresando al modelo geocéntrico, la inteligencia ha venido de la mano con la mente consciente. Así como otras cualidades (como la voluntad, la motivación, la toma compleja de decisiones, etc.) queson los pilares de una comunidad tecnológica. Además, si buscamos a nuestros similares en el universo, tendrían estos que tener mente consciente. Pues es este fenómeno el que nos hace humanos.

A no ser que se piense que se pueda dar un gran salto en inteligencia sin la mediación de una mente consciente -que siquiera le dé un impulso inicial, como pasa con los desarrollos actuales de la Inteligencia Artificial que necesitan humanos conscientes que los programen y construyanpodríamos llegar a creer que seres gregarios como las hormigas o abejas, que tienen mentes inconscientes, puedan llegar a formar una comunidad tecnológica. Algo a mi parecer muy improbable o hasta imposible.

Además, el fenómeno de la consciencia ha venido de la mano con el fenómeno de la vida, ha surgido de ella. La vida, como acumuladora de información, proporcionó la complejidad necesaria para que surjan mentes conscientes. $Y$ es que para que se formen seres conscientes e inteligentes, es necesario que la materia pase cierto umbral de complejidad a través de niveles de organización.

Haciendo un esquema que represente lo que paso en la Tierra, vemos que: 


\section{Átomo de CARBONO $\rightarrow \quad$ VIDA $\rightarrow$ MENTES $\rightarrow$ CONCIENCIA $\rightarrow$ INTELIGENCIA $\rightarrow$ COMUNIDAD TECNOLOGICA}

\section{En esta cadena de sucesos hay al menos tres cuellos de botella o eventos que son sumamente improbables. Fuera de estos eventos, los demás se desencadenarían naturalmente con toda probabilidad.}

\section{Primer cuello de botella: la vida}

El paso de muchas macromoléculas complejas a lo que Richard Dawkins llamaría el "primer reproductor", antecesor de la unidad de estructural de vida (la célula), es el primer evento improbable que identificamos. Esa molécula, capaz de hacer copias de sí misma usando los recursos del entorno, podría haber sido algún tipo de retrovirus con ARN. Pero una vez que aparece, se desencadenaría -con mucha más probabilidad- los organelos intracelulares y luego la célula en sí, mediante un proceso de competencia o evolución (Dawkins, 1993). Llenando al planeta de un sinfín de organismos similares a las bacterias. Este es el tipo de vida o proceso que los astrobiólogos esperan encontrar en otros planetas.

Antes de que se formen las macromoléculas, los físicos sostienen que son necesarios átomos de elementos más pesados que el hidrógeno, el elemento más abundante en el universo, que provendrían de restos de supernovas (Bachiller, 2015). En efecto, ya hemos visto que el Átomo de Carbono es el candidato ideal para general complejidad molecular.

\section{ATOMO DE CARBONO $\rightarrow$ MACROMOLECULAS $/ / \rightarrow / /$ PRIMER REPRODUCTOR $\rightarrow$ ORGANELOS INTRACELULARES $\rightarrow$ CELULA}

En la tierra este paso se dio, aproximadamente, mil millones de años después que se formó el planeta.

\section{Segundo cuello de botella: la pluricelularidad animal}

El paso de organismos unicelulares a cuerpos pluricelulares (específicamente animales) es el segundo evento improbable que identificamos. Gracias a este cuello de botella, los astrobiólogos esperan encontrar, con más probabilidad, vida o rastros de vida -similar a la unicelular- en otros planetas o entornos extraterrestres.

La multicelularidad ha aparecido de varias veces y de manera independiente a lo largo de la historia de la vida, especialmente de forma agregativa como es el caso de la eucariota Dictyostelium discoideum. Este es un protozoo que es unicelular durante la mayor parte de su ciclo biológico, pero -para reproducirse- forma agregados de células distintas genéticamente que se organizan en un pedúnculo reproductor.

La pluricelularidad que nos interesa, aquella que permitirá pasar al siguiente nivel de complejidad creando un organismo multicelular estable, es la de tipo clonal. En este tipo de pluricelularidad todas las células del agregado compartirán el mismo material genético, o sea serán clones (Alex de Mendoza, 2013). 


\section{CELULA $\rightarrow$ PLURICELULARIDAD $/ / \rightarrow / /$ PLURICELULARIDAD CLONAL $\rightarrow$ NEURONA $\rightarrow$ MENTES}

Este paso se produjo -en la Tierra- hace 500 millones de años, aproximadamente 3 mil 500 millones de años despuésde la aparición de la vida.

\section{Tercer cuello de botella: la mente consciente}

El paso de los animales con mentes inconscientes a animales con mentes conscientes es el tercer evento improbable que identificamos. Es tal vez el más improbable de los tres eventos que analizamos, pues la vida se maneja muy bien sin un "Yo". Es un lujo tener una mente consciente (y más aún como la humana).

\section{MENTES $/ / \rightarrow / /$ MENTE CONSCIENTE $\rightarrow$ INTELIGENCIA Y VOLUNTAD $\rightarrow$ COMUNIDADES TECNOLOGICAS}

Este paso se dio -con casi total seguridad- con la ascensión de los mamíferos. Se debate si los reptiles y las aves (que son prácticamente reptiles emplumados) poseen mente consciente, pero se sabe poseen las sensaciones básicas con mucha seguridad. No todas las mentes conscientes son "igual de conscientes", es obvio que hay una gradualidad de menor a mayor que no solo se da de especie a especie (el humano tiene la mente consciente más compleja de todas las otras especies, incluso que la de los otros homínidos extintos) sino que también se da de individuo a individuo.

Sostengo que las comunidades tecnológicas surgen con naturalidad cuando las mentes conscientes se gregarizan. El mismo proceso de gregarización provoca la complejidad de las mentesen un bucle de realimentación positiva. A medida que los grupos van creciendo en unidades "conscientes", es necesaria la sofisticación de los procesos que mantienen estables a la sociedad de mentes conscientes: una tecnología surge de manera natural.

IV. La ecuación de Drake y una tentativa de reformulación

"La Ecuación de Drake fue concebida con la intención de estimar el número de civilizaciones con capacidad radial (...) que existirían en nuestra Galaxia (...):

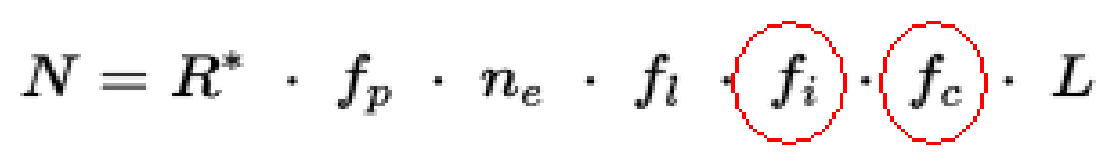

Donde $R$ representa la tasa de formación de estrellas en la Vía Láctea. El Factor $f_{p}$ corresponde a la fracción de estrellas que tienen planetas en su órbita. $N_{e}$ es el número de esos planetas orbitando dentro de la ecosfera de la estrella. El resto de factores $f$ corresponden a la fracción de planetas, similares a la Tierra, en donde se ha desarrollado vida ( $\left.f_{1}\right)$, en donde se ha desarrollado vida inteligente $\left(f_{i}\right)$ y en los que existen civilizaciones con capacidad radial $\left(f_{c}\right)$. Por último $L$ representa el tiempo de supervivencia que alcanza una civilización con capacidad de comunicación por ondas radiales. Lamentablemente la mayor parte de estos factores tienen valores desconocidos" (Avendaño, 1999. p. 72) 
Los parámetros quinto y sexto, que están en círculo rojo, son aquellos que podríamos cambiar por estos otros, teniendo en cuenta los 3 cuellos de botella vistos anteriormente:

f, seguiría representado la fracción de planetas de la zona habitable (ecosfera) donde se ha desencadenado algún proceso similar a la vida.

\section{$f_{\mathrm{i}}$ se cambiaría por $f_{\mathrm{pl}}$}

Donde $\mathbf{f}_{\mathrm{pl}}$ representaría la fracción de los planetas con vida donde los seres vivos consiguieron algo similar a la pluricelularidad clonal animal.

\section{Se añadiría un nuevo parámetro $f_{m c}$}

Donde $\mathbf{f}_{\mathbf{m c}}$ representaría la fracción de los planetas con vida donde los seres vivos consiguieron la mente consciente.

$f_{\text {c }}$ se reformularía como la fracción de los planetas con vida consciente que desarrollaron una comunidad tecnológica. Tal vez hasta podría eliminarse, si tomamos en consideración que la mente consciente desencadenaría naturalmente -y con mucha probabilidad- algo similar a una comunidad tecnológica.

\section{Conclusiones}

La llamada búsqueda astrobiológica -o búsqueda de vida fuera de la Tierra- debería tener como finalidad ultima la búsqueda de seres con mentes conscientes en el Universo, pues son ellos en realidad nuestros similares, teniendo en cuenta la principal característica que nos hace personas humanas. De estos seres sacaríamos la mayor cantidad de información útil.

Sin embargo, no debe bajarse la guardia en la búsqueda de los otros procesos, como la vida, que hicieron posible la acumulación de la información o complejidad necesaria para la aparición de la mente consciente. La mente consciente sólo puede surgir cuando el nivel de complejidad ha superado un umbral bastante alto.

La llamada búsqueda astrobiológica usa criterios altamente especulativos, pues es mucho lo que se desconoce del entorno extraterrestre. Sin embargo, como disciplina científica, debe extrapolar los conocimientos (de cómo se acumuló la complejidad en la Tierra) hacia ese entorno exterior y eso es lo que se llama "paradigma geocéntrico", lo cual no es nada criticable.

En el análisis de cómo evolucionó la mente consciente encontramos tres eventos improbables, que hemos denominado "cuellos de botella". Éstos se han dado secuencialmente y son: la aparición de la vida, la aparición de la multicelularidad clonal y la aparición de la menteconsciente. De todos ellos creo que la aparición de la mente consciente es el más improbable por su propia naturaleza.

Los tres eventos improbabilísimos deben plasmarse en cualquier ecuación que intente calcular la cantidad de comunidades tecnológicas en la galaxia o en el universo o las probabilidades que ellas existan. 


\section{Referencias}

Alex de Mendoza, A. S. (2013). El Origen de la Multicelularidad. Investigacion y Ciencia, 32-39. Avendaño, M. P. (1999). ¿Estamos solos en el Universo? Charlas de Fisica- Universidad de Tarapaca , 71-79.

Bachiller, R. (2015). El origen astroquimico de los sistemas planetarios y la vida. Investigacion y Ciencia, 46-54.

Bartra, R. (2005). Antropologia del Cerebro. Mexico: Fondo Cultura Economica.

Bernardo, H. (2007). ¿Que es la vida? Un problema epistemologico. Recuperado el 3 de Junio de 2016, de Más allá de A Parte Rei: http://serbal.pntic.mec.es/ cmunoz11/bernardo33.pdf

CAPPELLETTI, Á. (1981). Sobre el Infinito Universo y los Mundos, Traduccion de Giordano Bruno. Buenos Aires: Aguilar.

Damasio, A. (2006). En busca de Spinoza. Barcelona: Drakontos.

Damasio, A. (2010). Y el Cerebro creo al Hombre. Barcelona: Destino.

Dawkins, R. (1993). El gen Egoista. Barcelona: Salvat Editores.

Dawkins, R. (2009). Evolucion: el mayor espectaculo sobre la tierra. Madrid: Espasa.

Duve, C. d. (1995). Vital Dust: Life As a Cosmic Imperative. Londres.

Goldstein, E. (1999). Sensación y percepción. Mexico: International Thomson Editores.

Guillermo Gonzalez, J. R. (2006). El Planeta Privilegiado. Madrid : Ediciones Palabra.

Hawkins, J. (2005). Sobre la Inteligencia. Madrid: Espasa.

Houser, M. (2012). El origen de la mente. Investigacion y Ciencia, 37-41.

LeDoux, J. (1999). El cerebro emocional. Buenos Aires: Planeta Argentina.

Matej Hochel, E. G. (2002). La consciencia. Granada: Editorial debate.

Ponzio, P. (2006). El concepto del yo en la metafísica moderna del Siglo XVII: Campanella y Descartes. Topicos Revista de filosofia de Santa Fe.

Sagan, C. (1994). La busqueda de vida extraterrestre. Investigacion y Ciencia, 63-69.

Schrodinger, E. (2005). ¿Que es la Vida? Salamanca-España: Textos de Biofisica.

Waal, F. d. (2009). La edad de la Empatia. Mexico: Tusquets Editores Mexico.

Wade, L. C. (2012). Quimica Organica. Madrid: Pearson.

Zimmer, C. (2008). Genetica de la inteligencia. Investigacion y Ciencia, 44-51. 